NASA Technical Memorandum 102080

AIAA-89-1980

\title{
Conservative Treatment of Boundary Interfaces for Overlaid Grids and Multi-level Grid Adaptations
}

Young J. Moon

Sverdrup Technology, Inc.

NASA Lewis Research Center Group

Cleveland, Ohio

and

Meng-Sing Liou

National Aeronautics and Space Administration

Lewis Research Center

Cleveland, Ohio

Prepared for the

Ninth Computational Fluid Dynamics Conference

sponsored by the American Institute of Aeronautics and Astronautics Buffalo, New York, June 13-15, 1989

\section{N/SA}


Conservative Treatment of Boundary Interfaces

for Overlaid Grids and Multi-level Grid Adaptations

\author{
Young J. Moon ${ }^{\dagger}$ \\ Sverdrup Technology, Inc. \\ NASA Lewis Research Center Group \\ Cleveland, Ohio 44135 \\ and \\ Meng-Sing Liou ${ }^{\ddagger}$ \\ National Aeronautics and Space Administration \\ Lewis Research Center \\ Cleveland, Ohio 44135
}

\begin{abstract}
Conservative algorithms for boundary interfaces of overlaid grids are presented. The basic method is zeroth order, and is extended to a higher order method using interpolation and subcell decomposition. The present method, strictly based on a conservative constraint, is tested with overlaid grids for various applications of unsteady and steady supersonic inviscid flows with strong shock waves. The algorithm is also applied to a multi-level grid adaptation in which the next level finer grid is overlaid on the coarse base grid with an arbitrary orientation.
\end{abstract}

\section{Introduction}

Unstructured grid algorithms [1-3] have been popular in recent years, because they have flexibility in grid topology and also have efficiency in grid adaptation, in comparison with structured grid algorithms. However, the main drawback of the unstructured grid algorithms lies in the limitation of selecting the time integration scheme: one has to use an explicit or point-wise implicit algorithm. A general motivation of the present study is to develop a counterpart of the unstructured grid algorithms for structured grids. This counterpart must not only have the geometric flexibility and grid adaptation efficiency of the unstructured grid algorithm, but also must have a freedom of choice in a variety of existing implicit schemes.

To be specific, the present methodology use the overlaid grid (Chimera technique) [4-5] to improve geometric flexibility, and the multi-level grid adaptation $[6,23]$ for efficient grid adaptation. Multi-level grid adaptation here is not the mesh embedding technique

\footnotetext{
$\dagger$ Research Engineer, Member AIAA.

$\ddagger$ Chief, Computational Fluid Dynamics Branch, Member AIAA.
}

in which coarse grid cells are locally refined by embedding the next-level finer subcells. Instead, a next-level structured grid block is generated overlaying on the coarse base grid with an arbitrary orientation. This approach has the following merits. First, it gives geometrical independence between grid levels. Second, it allows natural alignment and clustering between grids and features in the solution. This grid alignment with the solution feature (e.g, shock or contact surface) possesses an extra advantage in conjunction with characteristic-based shock capturing shemes such as the flux-difference splitting [7] or flux-vector splitting [8] schemes. Since these numerical schemes are, in general, based on a one dimensional theory, if a shock or contact discontinuity is obliquely aligned with the grid cell (typically in a two dimensional case), the discontinuity cannot be numerically captured in fewer cells as when the discontinuity is aligned with the grid.

Overlaid grid and multi-level grid adaptation will introduce blocks of structured grids which can overlay each other with an arbitrary orientation. Then each block of the structured grid is solved by an implicit scheme using an ADI or LU algorithm. This approach, which is generally defined as a domain decompostition method, is also naturally suited for parallel processing.

However, the success of the overlaid grid and multilevel grid adaptation techniques relies on correct communication among overlaid grids through boundary interfaces. Previous studies on this problem can be catagorized into two groups: (i) interpolation of the neighboring variables $[4,5,9]$, and (ii) characteristicstype approach [10-12]. Since neither of them necessarily satisfies any form of a conservative constraint, the solutions on overlaid grids are often mismatched with each other. This may result in instablility or oscillations, especially when a shock wave passes through boundaries of overlaid grids. Nonetheless, characteristics-type approaches show improvements in accuracy and convergence over interpolation methods.

The main constraint in updating boundary interface variables is that any valid procedure must ensure conservative properties. For the generally overlaid grid 
system, Berger [13] attempted to derive a conservative, finite difference flux scheme. However, the stencil of fluxes becomes irregular and consequently raises difficulties in its extension, not only to three dimensions but also to implicit implementations (e.g, extra Jacobians emerge with consequent restructuring of the left hand side matrix). In the early work, Rai [14] applied a conservative procedure to patched grid systems where the interface between two patch boundaries becomes a line for two dimensions. In this case global conservation can be implemented as follows: balance of the temporal surface fluxes and continuity requirment of the dependent variables at the patch interface. This approach has been extended to three-dimensional patched grids [1719] where the interface between grid patches becomes a plane. The basic idea is to obtain the unknown surface fluxes by interpolation with the area-weighted coefficients, enforcing a global conservative constraint.

In the present study with overlaid grids, we apply a conservative constraint to an arbitrary domain in the region of overlap such that the conserved quantities such as the mass, momentum, and total energy are preserved in the domain at a particular time. Similar ideas have been used in the Lagrangian hydrodynamics $[15,16]$ for the rezoning process (transfer of solutions from an old grid to a new grid). Under the conservative constraint, we interpolate the unknown dependent variables $Q$ in the domain by area-weighting. We also extend the basic method (zeroth order) to a higher order algorithm by reconstructing the given piecewise constant solution, cell-by-cell, under the restriction of conservative constraint (volumetric conserved quantities in a cell). Application of the higher order method becomes important, especially when the data are transfered from coarse cells to fine cells.

In the following sections, we describe the governing equations, basic solver algorithms, and conservative algorithms for general overlaid grids. A number of applications are presented for the unsteady and steady inviscid problems with overlaid grids. Finally, this conservative procedure is applied to a multi-level grid adaptation.

\section{Governing Equations and Solver Algorithms}

The integral form of the time-dependent compressible Euler equations can be written in two dimensions as

$$
\frac{\partial}{\partial t} \iint_{A} Q d A+\int_{S} \hat{F} \cdot \hat{n} d S=0
$$

$Q=(\rho, \rho u, \rho v, e)^{T}$ is a vector of conservative variables and the vector $\hat{F}$ denotes the flux of mass, momentum, and energy across the surface of the control vol- ume. Here $e$ represents the total energy per unit volume. Based on the finite volume method, equation (1) is semi-discretized by assuming that the cell-centered conserved variables are constant within a cell, and that the flux integral at cell surfaces is also approximated by an average value of the numerical flux and the surface length.

We follow the MUSCL approach and Van Leer's flux-vector splitting procedures in evaluating the numerical flux at the surfaces. To be specific, we first use upwind-biased interpolation of a certain set of variables to obtain the values at the cell surface. With the interpolated values, the split fluxes at the cell surface are then evaluated according to flux-vector splitting procedures. The scheme is fully upwinded, second order accurate or upwind-biased, third order accurate for the convective and pressure terms. More details on the scheme can be found in reference [8]. Here we would like to briefly remark on the convergence of the scheme. We found that convergence is sensitive to the choice of the interpolating variables in the MUSCL approach and to the choice of limiters as well. The best convergence is achieved with primitive variables and the diffusive min-mod limiter. In the case of conserved variables for interpolation and the compressive min-mod limiter, convergence is locked in a limit cycle.

In the present study, the fourth order Runge-Kutta time-stepping scheme is used for the unsteady calculations. For the steady state problems, the first order Euler implicit method is used with an ADI algorithm.

\section{Conservative Algorithms for Overlaid Grids}

Suppose we have two domains which are overlaid in a general manner (see figure 1). Let $Q 1$ and $Q 2$ represent sets of conservative variables for the domains 1 and 2, respectively. A particular section in the region of overlap is denoted by a domain $\Omega$.

At a particular time $t$, a conservative constraint over the domain $\Omega$ is represented by

$$
\int_{\Omega} Q 1(\mathbf{x}, t) d \Omega \equiv \int_{\Omega} Q 2(\mathbf{x}, t) d \Omega=C(t)
$$

where $Q$ is a function of time $t$ and space coordinates $x$. For the rest of this section, let a distribution of $Q 2$ over the domain $\Omega$ be known. Then a set of conserved quantities $C(t)$ (e.g, mass, momentums, and total energy) over the domain $\Omega$ is known at time $t$, according to the equation (2). In a discrete sense, two domains are represented by computational grids and the piecewise constant, discrete solution of $Q 2$ over the domain $\Omega$ is given. 
The basic idea is to obtain the unknown $Q 1$ in the domain $\Omega$ from the given $Q 2$ by interpolation with the area-weighted coefficients, enforcing the conservative constraint, equation (2). There are several important facts to keep in mind. First, equation (2) is only a constraint condition over the domain $\Omega$. Therefore we would like to apply the equation (2), cell-by-cell, so that the conservative constraint is satisfied automatically in a global sense. Second, the constraint condition concerns only the conserved quantities $C(t)$ over the domain $\Omega$ (e.g, a $Q 2$ cell), not the variation of the conservative variable $Q 2(x, t)$ within the domain. In other words, the conservative constraint is satisfied, as long as the conserved quantities are preserved over the domain $\Omega$. This leads to a freedom in reconstructing the variation of $Q 2$ within the cell for the purpose of achieving a higher order redistribution to $Q 1$. Here we designate redistribution as the process of determining the distribution of the unknown $Q 1$ over the domain $\Omega$, under the conservative constraint. Third, the accuracy of conserved quantities $C(t)$ obtained for the domain $\Omega$ was predetermined by the truncation error in the solution of the grid 2. This means that the accuracy of the transferred conserved quantities on grid 1 cannot be increased by increasing the order of the redistibution procedure. Consequently, the best possible solution which can be constructed on grid 1 by this conservative constraint is the solution of the grid 2 itself.

\section{Zeroth Order Redistribution}

Suppose the domain $\Omega$ corresponds to a single cell of coarse grid 1 and to composite pieces of cells of fine grid 2. A schematic is shown in figure 2. Given a piecewise constant distribution of $Q 2$ from the solution on grid 2, apply the conservative constraint over the domain $\Omega$. Then equation (2) yields, in a discrete form,

$$
Q 1_{j, k}=\sum_{i=1}^{i m a x}\left[Q 2_{l, m} \frac{\left(A_{o v}\right)_{l, m}}{A_{j, k}}\right] \quad i \in(l, m)
$$

where $(j, k)$ and $(l, m)$ represent cell indices for grid 1 and 2, respectively. Also index $i$ indicates each $(l, m)$ cell overlapped by the $(j, k)$ cell, and $i m a x$ is the total number of them. $A_{j, k}$ is the area of the $(j, k)$ cell, and $\left(A_{o v}\right)_{l, m}$ is the portion of the $(l, m)$ cell area overlapped by the $(j, k)$ cell. This situation corresponds to the case of transfering the data from the fine cells to a coarse cell. The conservative constraint itself is sufficient to uniquely determine the $Q 1$ variables. We call this procedure the zeroth order redistribution, since we use the given piecewise constant values of $Q 2$.

Conversely, suppose the domain $\Omega$ corresponds to cells of fine grid 1 and to a single cell of coarse grid 2 (see figure 3). This case corresponds the data transfer from a coarse cell to fine cells. Then the conservative constraint yields again, in a discrete form, the following:

$$
\sum_{i=1}^{i \max }\left[Q 1_{j, k}\left(A_{o v}\right)_{j, k}\right]=Q 2_{l, m} A_{l, m} \quad i \in(j, k)
$$

where index $i$ indicates each $(j, k)$ cell overlapped by the $(l, m)$ cell, and $\left(A_{o v}\right)_{j, k}$ represents the portion of the $(j, k)$ cell area overlapped by the $(l, m)$ cell. In this situation, the conservative constraint is only a necessary condition, but is not sufficient to uniquely determine the $Q 1$ variables (one equation with imax unknowns). The zeroth order redistribution will set $Q 1$ equal to $Q 2$ over the domain $\Omega$. Then equation (4) is automatically satisfied and the conservative constraint still holds. However, it yields an undesirable property such that the redistributed finer cells will represent a continous distribution of the coarse cells as a stairstep approximation (i.e, degrading smothness). This may have effects on stability and consequently on the convergence.

\section{Higher Order Redistribution}

In order to circumvent this problem, a higher order redistribution procedure is proposed here. Since equation (4) is an underdetermined system, we have freedom to assign a proper value to each overlapping $(j, k)$ cell, while a conservative constraint is maintained. The idea is based on a reconstruction of the given piecewise constant solution over a $Q 2$ cell with the conservative constraint.

It would be nice if we could represent the piecewise variation in a single, analytic form, with the conservative constraint still satisfied in a $Q 2$ cell (i.e., preserving the volumetric conserved quantities). For example, a linear approximation for the reconstructed $Q 2$ within a cell is expressed as an equation of a plane with a single free parameter which is determined by the conservative constraint. However, the only fact that matters is how many cells of grid 1 are occupying the grid 2 cell (i.e, area ratio between two grid cells), because, after all, the occupying grid 1 cells will be represented as a piecewise constant approximation. Therefore we propose to represent a variation within a $Q 2$ cell with a finite number of piecewise constant approximations.

If we have a coarse $(l, m)$ cell of grid 2 , then decompose it into multi-subcells denoted by index is. For example, decompose the $(l, m)$ cell into four subcells by connecting the midpoints of four surfaces of $(l, m)$ cell. A schematic is shown in figure 4. These four subcells are not necessarily to be matched with the fine cells of grid 1 in figure 3 , but it is important to keep the area ratio between grid 1 cells and the decomposed subcells 
close to unity. The best strategic decomposition is to mimic the orientation and size of the cells of grid 1.

Now obtain an interpolated value $\bar{Q}_{i}$, for each subcell $(l, m, i s)$. For example, $\bar{Q}_{i s=4}$ is a subcell-centered value obtained by triangular interpolation using its surrounding coarse cells, i.e, $Q 2_{l, m}, Q 2_{l-1, m}$, and $Q 2_{l, m+1}$ (see figure 4). Then we define a weighting coefficient $C_{i s}$ for each $(l, m, i s)$ subcell as follows

$$
C_{i s}=\frac{\tilde{Q}_{i s} A_{i s}}{\sum_{i d=1}^{4} \tilde{Q}_{i d} A_{i d}} \quad \text { is } \in(l, m)
$$

where $\tilde{Q}_{i,}=\bar{Q}_{i s}\left(Q 2_{l, m}\right), A_{i,}$ is a subcell area, and $i d$ represents a dummy index for subcell index is. The weighting coefficient is designed to be proportional to the product of the interpolated value and its area so that it relates to the conserved quantities, while it is scaled so that summation of all $C_{i}$, becomes unity.

Multiplying the volumetric conserved quantities $Q 2_{l, m} A_{l, m}$ by the weighting coefficient $C_{i s}$, finally reconstructs the piecewise constant $Q 2$ over the $(l, m)$ cell into four piecewise constant $\widehat{Q}_{i s}$ over the $(l, m, i s)$ subcells where $\hat{Q}_{i s}$ is expressed as

$$
\widehat{Q}_{i s}=\frac{\tilde{Q}_{i s}}{\sum_{i d=1}^{4} \tilde{Q}_{i d} A_{i d}}\left[Q 2_{l, m} A_{l, m}\right]
$$

Then the reconstructed subcell value $\widehat{Q}_{i}$, automatically satisfies the conservative constraint over the domain $\Omega$ (i.e, the $(l, m)$ cell) as

$$
\sum_{i s=1}^{4}\left[\widehat{Q}_{i s} A_{i s}\right]=Q 2_{l, m} A_{l, m}
$$

This concludes the process of reconstructing $Q 2$ over the domain $\Omega$ for the four subcell case.

We now generalize the above procedure to the case where the total number of subcells within the $(l, m)$ cell is ismax. Returning to the case described in figure 3 , equate the left hand sides of equations (4) and (7) to obtain

$$
\sum_{i=1}^{i \max }\left[Q 1_{j, k}\left(A_{o v}\right)_{j, k}\right]=\sum_{i s=1}^{i s \max }\left[\widehat{Q}_{i s} A_{i s}\right]
$$

If ismax $\geq$ imax, then the situation becomes such that the conservative constraint can uniquely determine the $Q 1$ variables:

$$
Q 1_{j, k}=\sum_{i s=1}^{\leq i s m a x}\left[\hat{Q}_{i,} \frac{\left(A_{o v}\right)_{i s}}{A_{j, k}}\right] \quad \text { is } \in(l, m)
$$

where $\left(A_{o v}\right)_{i *}$ represents the portion of $(l, m, i s)$ subcells overlapped by the $(j, k)$ cell.
Finally, the conservative constraint, equation (2), can be written in a general form for the higher order redistribution as

$$
Q 1_{j, k}=\sum_{i=1}^{i m a x}\left(\sum_{i s=1}^{\leq i s \max }\left[\widehat{Q}_{i, i s} \frac{\left(A_{o v}\right)_{i, i s}}{A_{j, k}}\right]\right)
$$

where $i$ indicates each overlapped $(l, m)$ cell and is indicates each overlapped subcell $(l, m, i s)$ within $(l, m)$ cell. The order of accuracy in this redistribution is dependent upon the selected order in the interpolation procedure and the total number of subcells, ismax, within an $(l, m)$ cell as well. However, if the upper limit of is equals to ismax in equation (10), then the equation (10) automatically becomes equation (3).

The reconstruction process described here can be viewed as the procedure of raising the order of accuracy of the initial-value interpolation in a "projection stage" [20] of a Godunov scheme. The higher order redistribution may create a non-physical solution (overshoot or undershoot) near discontinuities. Therefore a limiter is recommended for future application in the interpolation process. For all the test cases presented in this paper, the limiter was not necessary.

\section{Algorithm for Computing Area of Overlap}

The conservative algorithms described in the previous sections can be summarized as a rule of area weighting. The remaining question is how to evaluate the area of overlap between two arbitrarly shaped quadrilateral meshes. The algorithm should consist of logically simple procedures that are extendable to the three dimensional case (evaluating a volume of overlap).

The algorithm consists of two processes called "enclosure" and "crossing". "Enclosure" determines if a point is within a cell or not, and "crossing" calculates the crossing point between either a line with finite length or one with infinite length. Using these two processes, one can find the vertices of the area of overlap, which always form a convex polygon. The procedure consists of six possible combinations in forming the vertices of a polygon from the vertices and crossed points between two overlapping quadrilateral meshes. Finally the vertices of the polygon are arranged in a connecting order.

The area of the convex polygon is evaluated by

$$
A_{o v}=\frac{1}{2}\left|\sum_{i=1}^{n p}\left(x_{i+1} y_{i}-x_{i} y_{i+1}\right)\right|
$$

where $n p$ represents the total number of polygon surfaces. Here $\left(x_{i}, y_{i}\right)$ denotes a vertex point of a polygon. The most computationally intensive part of this algorithm lies in determining which cells of two grids share 
a common region. A knowledge-based tree search algorithm was used to improve the efficiency. This is a pre-processing procedure with a cost of memory overhead for the geometric information.

\section{Results and Discussion}

The conservative algorithm for overlaid grids, described in the previous section, was applied to various unsteady and steady problems of supersonic inviscid flows. In order to demonstrate the conservative property of the present method, all the problems possess strong moving or stationary shock waves which cross the boundary interfaces of the overlaid grids. Finally, this procedure was combined with multi-level grid adaptation to display the versatility of the algorithm.

Before discussing the results, some details of implementing the conservative interface treatments will be briefly described. Since the fully upwind second order or upwind-biased third order accurate scheme has a five point stencil, the scheme reduces to first order accuracy at the two cells next to a boundary. Therefore two cells at the boundary of grid 1 are intentionally placed away from the other two cells at the boundary of grid 2 (shown in figure 5 ). There are two reasons for this. First, the third cells from the boundaries of grid 1 and 2 can use the five point scheme to have at least second order accuracy. Second, the two boundary cells of grid 1 and 2 can be updated by the conservative algorithms using at least second order accurate solutions from each grid. This boundary update is explicitly conducted so that it can be easily applied to explicit or implicit schemes.

One last remark regards the synchronization of the solutions on both grids. The conservative constraint, equation (2), is valid at a particular time on both grids. Therefore, if two different time steps are used for each grid, one must ensure that the conservative boundary update is conducted at the same elapsed time, especially for the time accurate calculations. One way to synchronize is to interpolate the solution in time. For example, advance a solution on one grid with large time step. Then, interpolate the solution in time to a number of time levels necessary for the other grid solution to "catch up" to the advanced time. Finally apply the conservative algorithm at each sub-time level, using the timewise interpolated solution. The accuracy and stability of this procedure is not yet tested. For all the results shown in the present study, synchronization was achieved by advancing the solutions with the smaller time step between the two grids.

Time-dependent Calculations

\section{Case 1}

The conservative algorithm is tested for a timedependent, shock tube problem. Here we intentionally solve this one-dimensional problem in two dimensions. The computational domain is composed of overlaid grids: $45 \times 9$ and $55 \times 11$ meshes for grid 1 and 2, respectively (shown in figure 6 (a)). The lines between two overlaid grids are mismatched both horizontally and vertically. Pressure and density ratios of 10 are set at $x=2$ as an initial condition.

Figure 6 (b) displays the density contours at $t=0.61$, which are plotted on top of each other. The zeroth order redistribution matches the solutions well in the overlapped region, since the grid spacings of the two grids are comparable. A comparison of the solutions with the exact solution is shown in figure 6 (c). The solid line represents the exact solution (density distribution), and the open circles and the dashed line correspond to the solutions from grid 1 and 2 , respectively. Results show that the data communication through boundary interfaces between two overlaid grids was conducted conservatively and time-accurately.

Case 2

The same problem as case 1 is tested, but the grid spacing between the two grids is doubled in the $x$-direction: $41 \times 9$ and $81 \times 11$ meshes for grid 1 and 2, respectively (shown in figure 7 (a)). Results of the zeroth and higher order redistributions are compared in figure 7 (b) and (c). Figure 7 (b) (the zeroth order redistribution) shows the mismatched density contours from the two solutions in the overlapped region. Actually, the coarse grid solution is accurately obtained, because the data are transfered from fine cells to coarse cells. The difficulty is encoutered for the fine grid in which the situation is reversed. The stairstep contours can be observed in the figure 7 (b). The result of the higher order redistribution, shown in figure 7 (c), noticeably improves the solution of the fine grid in the overlapped region. For the results from the higher order redistribution presented in this paper, the coarse cells are decomposed into four subcells, according to midpoints of the coarse cell surfaces. As mentioned earlier, a more optimal subcell decomposition will lead to a better match of the solutions between two overlaid grids.

Case 3

A more realistic two dimensional unsteady interactions of a moving shock of $M_{s}=7.19$ in a $20^{\circ}$ bent channel were simulated on two overlaid grids, using the conservative algorithm with the higher order redistri- 
bution. Figure 8 (a) shows the two overlaid grids with $101 \times 27$ and $91 \times 31$ meshes. A moving normal shock of $M_{2}=7.19$ is set at the inlet of the $20^{\circ}$ bent channel as an initial condition.

This bent channel consists of convex and concave corners where expansion and compression of the induced supersonic flows $(M=1.7721)$ occur, respectively. In the lower compression region, a complexMach reflection, which is defined as a single Mach reflection with a kink near the triple point, is developing. In the upper expansion region, a Prandtl-Meyer fan is centered at the convex corner, and a normal shock wave is embedded between the fan and the incident shock wave to adjust the flow speed to the condition behind the diffracted part of the incident shock wave. Sequential plots of density contours (figures 8 (b) - (f)) display developments of the interactions among shock waves, expansion waves, and confined geometries. The solutions of two grids are well matched in the overlapped region. This result shows the capability of the present conservative algorithm for strong multiple shock waves crossing the boundaries of the overlaid grids.

\section{Steady-state Calculations}

\section{Case 1}

As a steady state application, the conservative algorithm was tested for a two dimensional oblique shock reflection on a flat plate. The free stream Mach number $M_{\infty}$ is 2.9 and the incident oblique shock angle is $29^{\circ}$. A uniform mesh of $61 \times 17$ is used for grid 1 , while grid 2 has a uniform mesh of $71 \times 15$ (figure 9 (a)). Figure 9 (b) shows a close-up view near the overlapped region. Mesh cells of each grid are mismatched in both $x$ and $y$ directions. Since the grid spacings between the two overlaid grids are comparable, the zeroth order redistribution was used. Figure 9 (c) displays pressure contours of each grid plotted on top of each other. The solutions from the two grids are well matched in the overlapped region. Two parallel solid lines indicate the boundaries of the overlapped region. The performance of the conservative algorithm for steady state problems is expected, since we iterate the unsteady solver to the steady state in a time marching manner.

\section{Case 2}

In this case, the incident oblique shock is simulated in the domain, $0 \leq x \leq 1.6$ and $0 \leq y \leq 1$. with the same physical flow conditions as the previous case. This time, the Rankine-Hougoniot conditions are applied at $(0,0)$. A uniform mesh of $41 \times 17$ is used for grid 1, while grid 2 has a uniform mesh of $81 \times 15$. The close-up view of the overlapped region is shown in fig- ure 10 (a). The grid spacing ratio between the two grids is $2: 1$ in the $x$ direction. Figure 10 (b) shows the pressure contours of the zeroth order redistribution. As discussed in the previous comparison for the unsteady case, stairstep contours of the fine grid can be observed in the lower portion of the overlapped region. Figure 10 (c) displays the pressure contours improved by the higher order redistribution.

\section{Case 3}

Supersonic flow with a free stream Mach number of $M_{\infty}=6.0$ over a blunt cylinder is tested with two overlaid grids. Grid 1 of $71 \times 30$ is placed next to the body, and grid 2 of $81 \times 35$ is overlaid on the grid 1 (shown in figure $11(\mathrm{a})$ ). Figure 11 (b) displays the details of grid lines in an upper portion of the overlapped region. The zeroth order redistribution is used in this case. Figure 11 (c) shows Mach number contours plotted on top of each other. The two solid lines indicate the boundaries of the overlapped region. Mach number contours of expanding flow from low subsonic to moderate supersonic are well matched in the overlapped region between the two grids. The surface pressure distribution is also compared with experiment in figure 11 (d), and agrees well with it.

Case 4

Edney's type III shock-on-shock interaction on a blunt cowl lip was tested with two overlaid grids. Supersonic flow with a free stream Mach number of $M_{\infty}=$ 6.0 over a blunt cowl lip is intersected at $\theta=14.4^{\circ}$ by an oblique shock inclined to the free stream at an angle of $22.67^{\circ}$. More details on the flow conditions can be found in [21]. The grid 1 of $89 \times 39$ is placed next to the body, and grid 2 of $99 \times 49$ is overlaid on grid 1 (figure 12 (a)). Figure 12 (b) shows a close-up view of the upper portion of the overlapped region. For this case, the zeroth order redistribution is also used. Figure 12 (c) shows Mach number contours plotted on top of each other. The overlapped region encloses interacting shocks, a triple point, shear layer, and expanding flows. Two solid lines indicate the boundaries of the two overlaid grids. Mach number contours of the two overlaid grids are well matched. The surface pressure distribution is also compared with experiment in figure 12 (d), and agrees relatively well, except near the shear layer impingement region. Better agreement would be obtained not only by solving the Navier-Stokes equations with the proper turbulence model for this turbulent shear layer [22], but also by refining the meshes of the two grids in the region near the impinging shear layer. 


\section{Multi-level Grid Adaptation}

The present conservative algorithm has a special application to multi-level grid adaptation for achieving high accuracy. A major difference from the work of Berger [23] is the use of next level finer grids which can be overlaid on the base grid with an arbitrary orientation.

Briefly describing the adaptation procedures, first obtain a solution converged to a certain level (e.g, trucation level) on a relatively coarse grid. Then, based on an error estimator or scanning of the flow with a proper detection criterion, generate a next level grid which is clustered and aligned with the solution features such as a shock wave, contact surface, boundary layer, or shear layer. On this grid, the coarse grid solution is interpolated by using the conservative algorithm. Then solutions of the base and next level grids will be advanced by communication of data through boundary interfaces.

As a demonstration of the above approach, case 2 in the steady state calculations was selected for grid adaptation. Figure 13 (a) shows a single uniform grid of $41 \times 41$ for a domain $(0 \leq x \leq 2.5$ and $0 \leq y \leq 2)$. Pressure contours are shown in figure 13 (b). Figure 13 (c) displays a pressure distribution at $y=0.5$. The oblique shock is numerically captured within appoximately 7 to 8 cells. This is a typical result of Van Leer's flux-vector splitting MUSCL scheme (upwind-biased third order accurate) when a shock wave lies obliquely to the grid lines, since the scheme is based on the one-dimensional theory.

A next level grid of 33x15 mesh is generated by scanning the maximum pressure gradient points from the base grid solution. Figure 14 (a) shows the grid which is clustered and aligned with the shock. Pressure contours from the refined solution obtained on the next level adapted grid is shown in figure 14 (b). Figure 14 (c) displays the pressure distributions at three different locations. As predicted [8] for a one dimensional case, the shock is numerically captured within two cells for all three locations. This grid alignment is one way of achieving the full quality of upwind TVD schemes, whereas the rotationally biased upwind scheme [24] has shown a similar performance.

\section{v. Conclusions}

Conservative algorithms for boundary interfaces of overlaid grids have been presented. The test results show the potential of the present method in various applications for unsteady and steady supersonic flows with strong shock waves. The proposed higher order redistribution presents a distinct improvement over the zeroth order redistribution for cases where the data are transfered from coarse cells to fine cells with the ratio of grid spacing between two overlaid grids no less than $2: 1$. The present conservative algorithm has a direct application to the domain decomposition method in which overlaid grids and multi-level grid adaptation are currently considered. It is believed that implementation of parallel processing for this multi-block domain strategy is an immediate next step.

\section{References}

[1] Peraire, J., Vahdati, M., Morgan, K., and Zienkiewicz, O., "Adaptive Remeshing for Compressible Flow Computations," J. Comp. Phys., Vol. 77, 1987.

[2] Mavriplis, D. J., “Accurate Mutigrid Solutions of the Euler Equations on Unstructured and Adaptive Meshes," AIAA Paper 88-3707, First National Fluid Dynamics Congress, Cincinnati, July 1988.

[3] Shapiro, R. A. and Murman, E. M., "Adaptive Finite Element Methods for the Euler Equations," AIAA Paper 88-0034, Reno, Jan. 1988.

[4] Dougherty, F. C., Benek, J. A., and Steger, J. L., "On Applications of Chimera Grid Schemes to Store Separation," NASA TM 88193, Oct. 1985.

[5] Benek, J. A., Donegan, T. L., and Suhs, N. E., "Extended Chimera Grid Embedding Scheme with Application to Viscous Flows," AIAA Paper 87-1126, AIAA 8th CFD Conference, Hawaii, July 1987.

[6] Berger, M. and Oliger J., "Adaptive Mesh Refinement for Hyperbolic Partial Differential Equations," J. Comp. Phys., Vol. 53, 1987.

[7] Roe, P., “Approximate Riemann Solvers, Parameter Vectors, and Difference Schemes," J. Comp. Phys., Vol. 43, 1981.

[8] Van Leer, B., "Flux-Vector Splitting for the Euler Equations," Lecture Notes in Physics, Vol. 170, 1982, pp. 507-512.

[9] Atta, E., "Component-Adaptive Grid Interfacing," AIAA Paper 81-0382, St. Louis, Jan. 1981.

[10] Eberhardt, S., "Overset Grids in Compressible Flow," AIAA Paper 85-1524, 1985.

[11] Bush, R. H., "External Compression Inlet Predictions using an Implicit, Upwind, Multiple Zone Approach," AIAA Paper 85-1521, 1985.

[12] Cambier, L. and Veuillot, J., "Computation of Casacade Flows at High Reynolds Number by Numerical Solution of the Navier-Stokes Equations," AIAA 
Paper 88-0364, Reno, Jan 1988.

[13] Berger, M., "On Conservation at Grid Interfaces," SIAM J. Numer. Anal., Vol. 24, No. 5, Oct. 1987.

[14] Rai, M. M., "A Conservative Treatment of Zonal Boundaries for Euler Equation Calculations," AIAA Paper 84-0164, 1984.

[15] Dukowicz, J., "Conservative Rezoning (Remapping) for General Quadrilateral Meshes," J. Comp. Phys., Vol. 54, 1984.

[16] Ramshaw, J., "Conservative Rezoning Algorithms for Generalized Two-Dimensional Meshes," J. Comp. Phys., Vol. 59, 1985.

[17] Hessinius, K., and Rai, M., "Three Dimensional, Conservative, Euler Computations Using Patched Grid Systems and Explicit Methods," AIAA Paper 86-1081, 1986.

[18] Kathong, M., Smith, R., and Tiwari, S., "A Conservative Approach for Flow Field Calculations on Multiple Grids," AIAA Paper 88-0224, 1988.

[19] Walters, R., Reu, T., Thomas, J., and McGrory, W., "A Longittudinally-Patched Grid Approach with Applications to High Speed Flows," AIAA Paper 88$0715,1988$.

[20] Van Leer, B., "Upwind-Difference Methods for Aerodynaime Problems Governed by he Euler Equations," AMS publications, Lectures in Applied Mathematics, Vol. 22, 1985.

[21] Moon. Y. J., "Interaction of an Oblique Shock Wave with Supersonic Flow Past a Blunt Body," Ph.D Dissertation, University of California, Berkeley, June 1988.

[22] Keyes, J. W., "Correlation of Turbulent Shear Layer Attachment Peak Heating near Mach 6," AIAA Journal, Vol. 15, Dec. 1977, pp. 1821-1823.

[23] Berger, M., "Adaptive Finite Difference Methods in Fluid Dynamics," von Karman Institute for Fluid Dynamics, Lecture Series 1987-04, 1987.

[24] Davis, S., "A Rotationally Biased Upwind Difference Scheme for the Euler Equations," J. Comp. Phys., Vol. 56, 1984. 


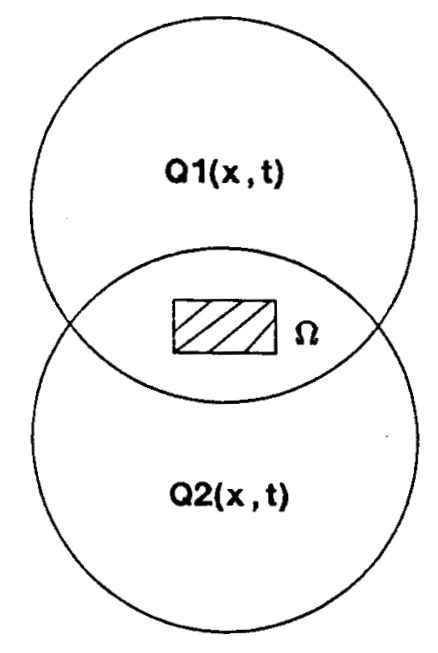

Fig. 1 Schematic of two overlaid grids

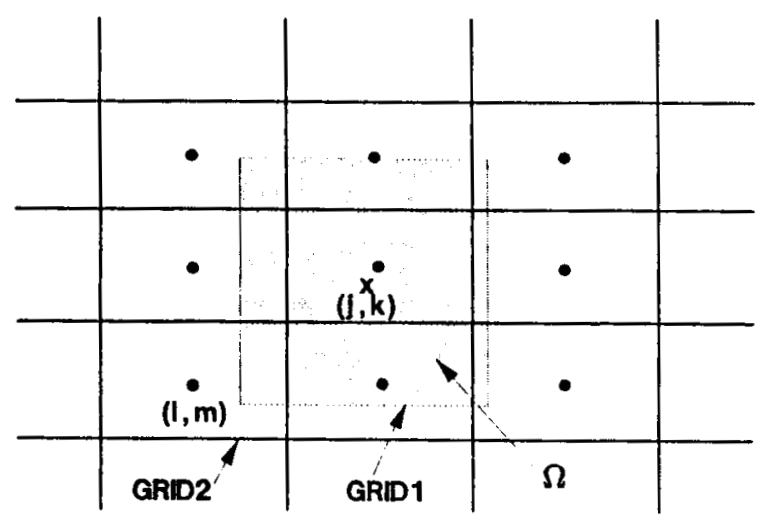

Fig. 2 Schematic of a uniquely determined system (fine to coarse)

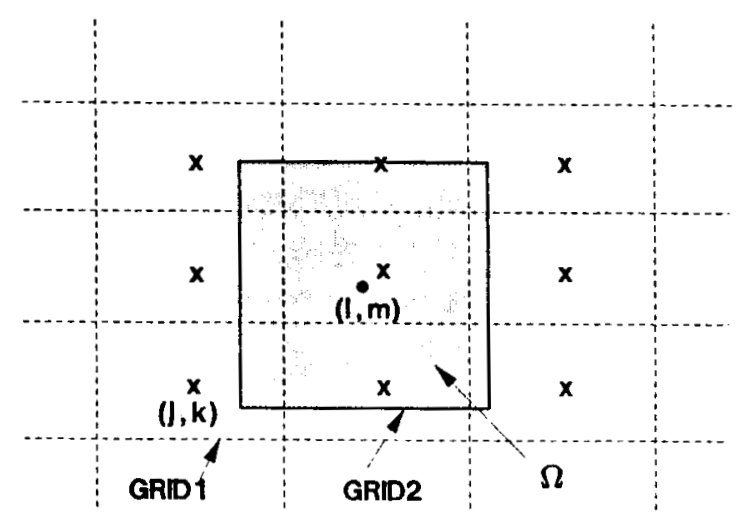

Fig. 3 Schematic of an underdetermined system (coarse to fine)

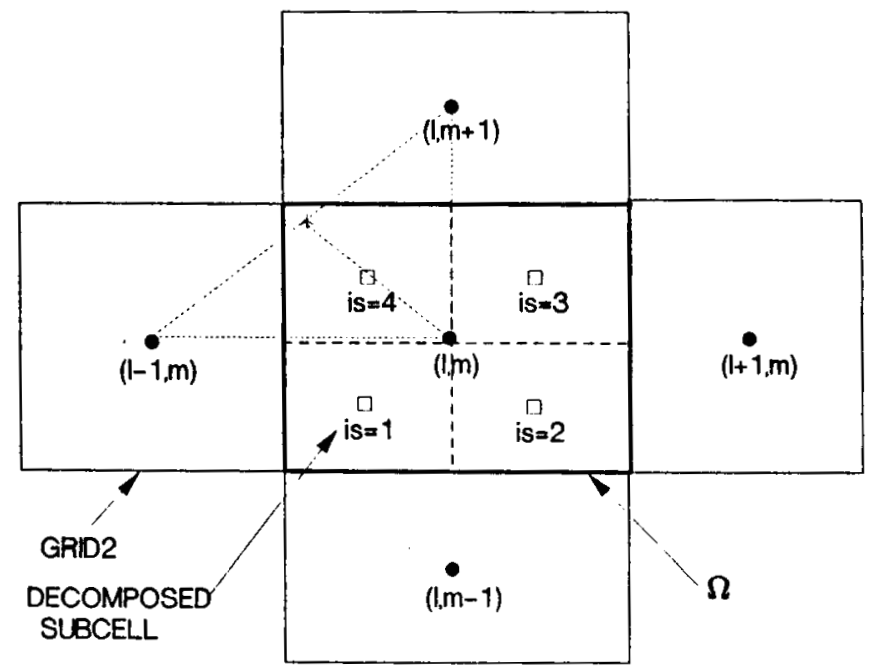

Fig. 4 Schematic of subcell decomposition and triangular interpolatior

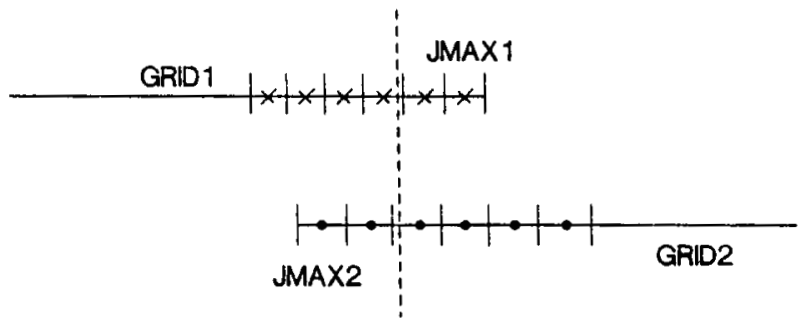

Fig. 5 Schematic of boundary interfaces layout 


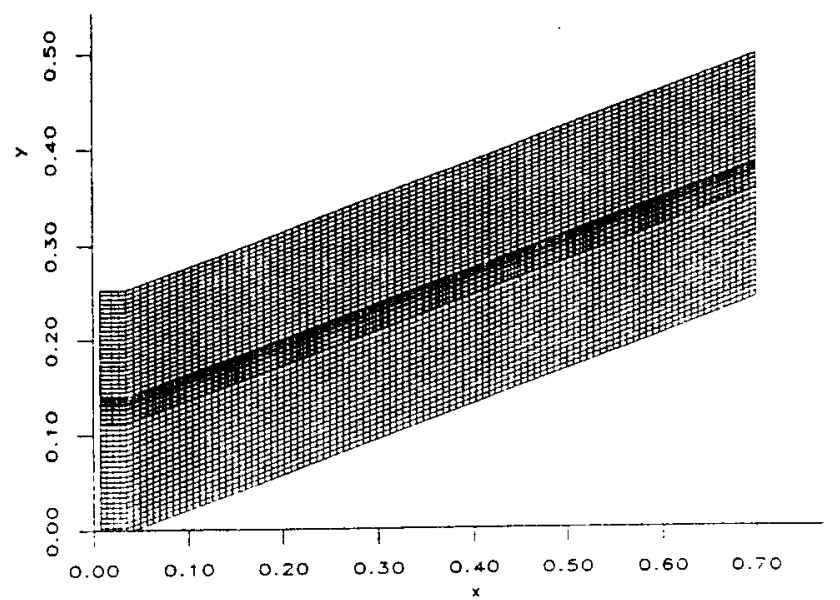

(a)

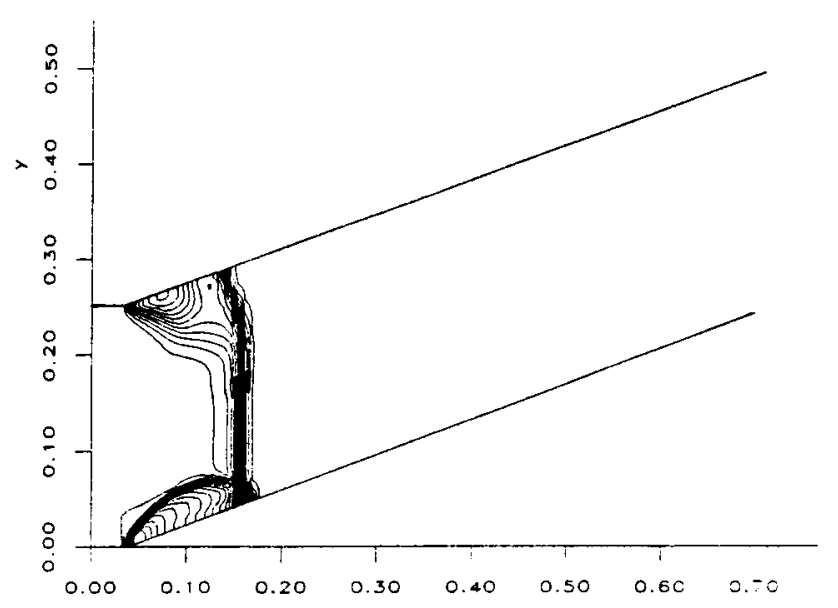

(b)

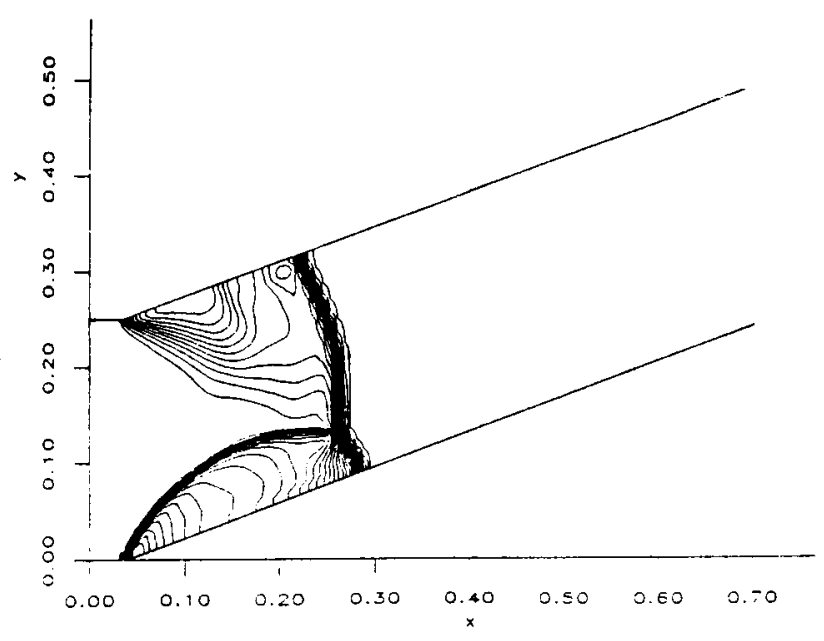

(c)

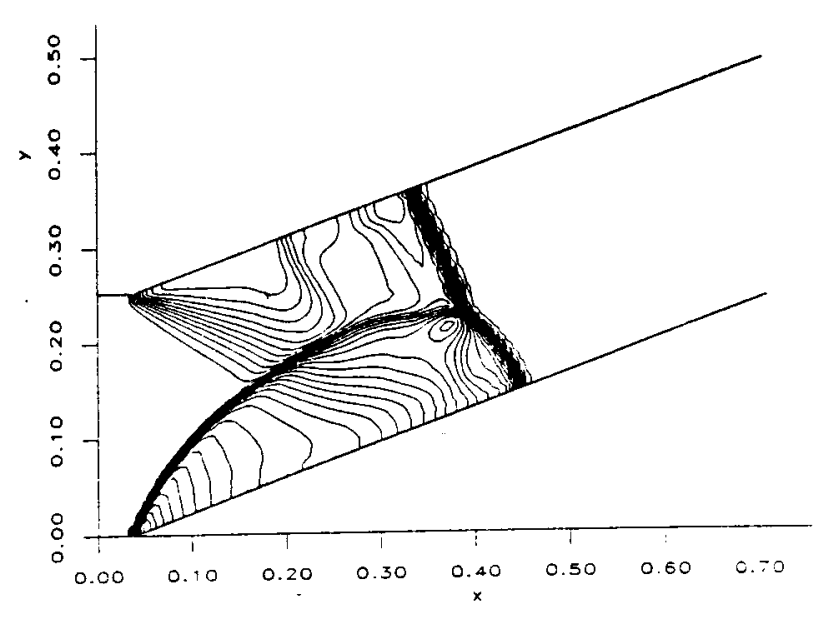

(d)

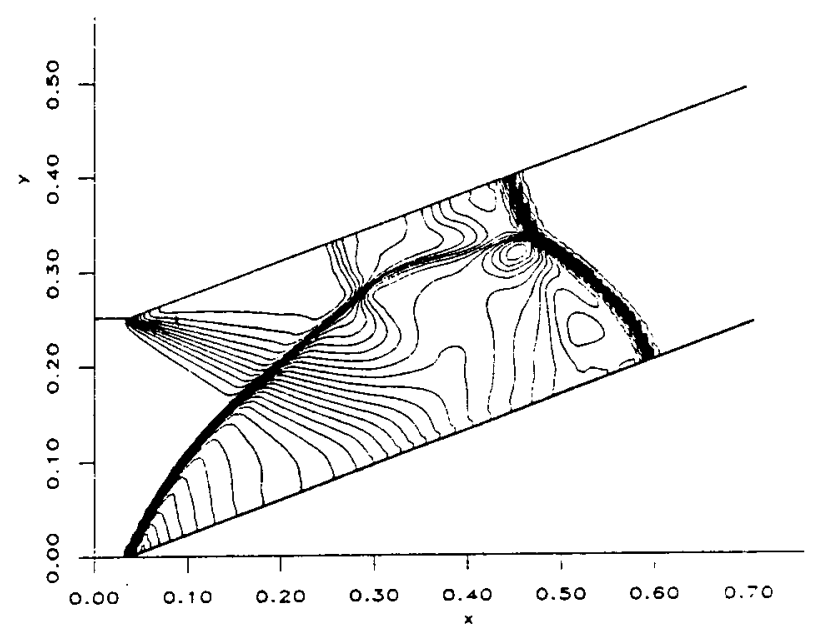

(e)

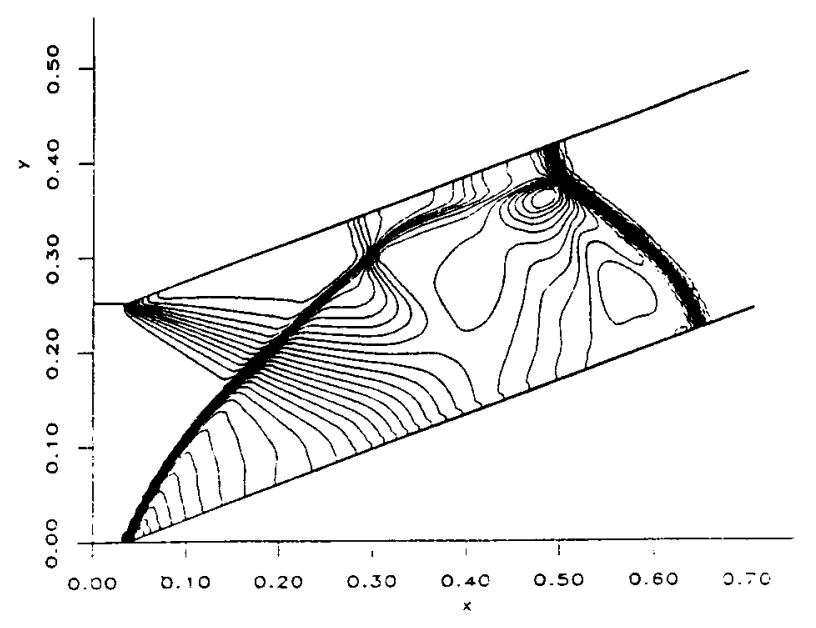

(f)

Fig. 8 (a) $20^{\circ}$ bent channel: grid 1 (101x27), grid 2 (91x31), (b)-(f) Density contours of moving shock interactions at five sequential stages (higher order redistribution). 


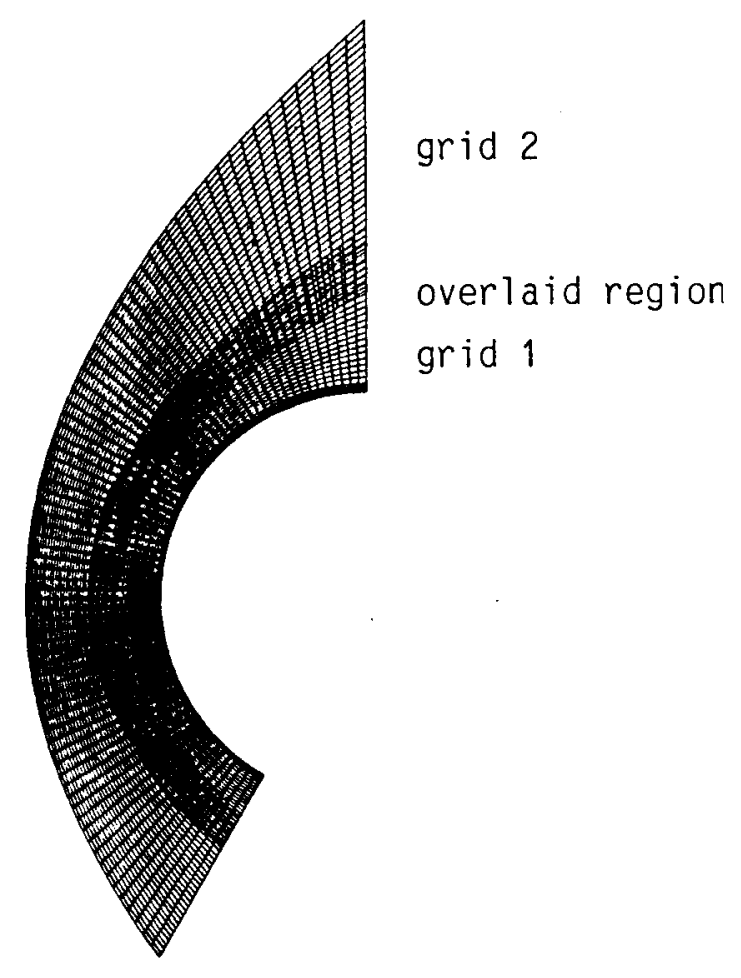

Fig. 11 (a) Supersonic blunt body flow: grid $1(71 \times 30)$, grid $2(81 \times 35)$

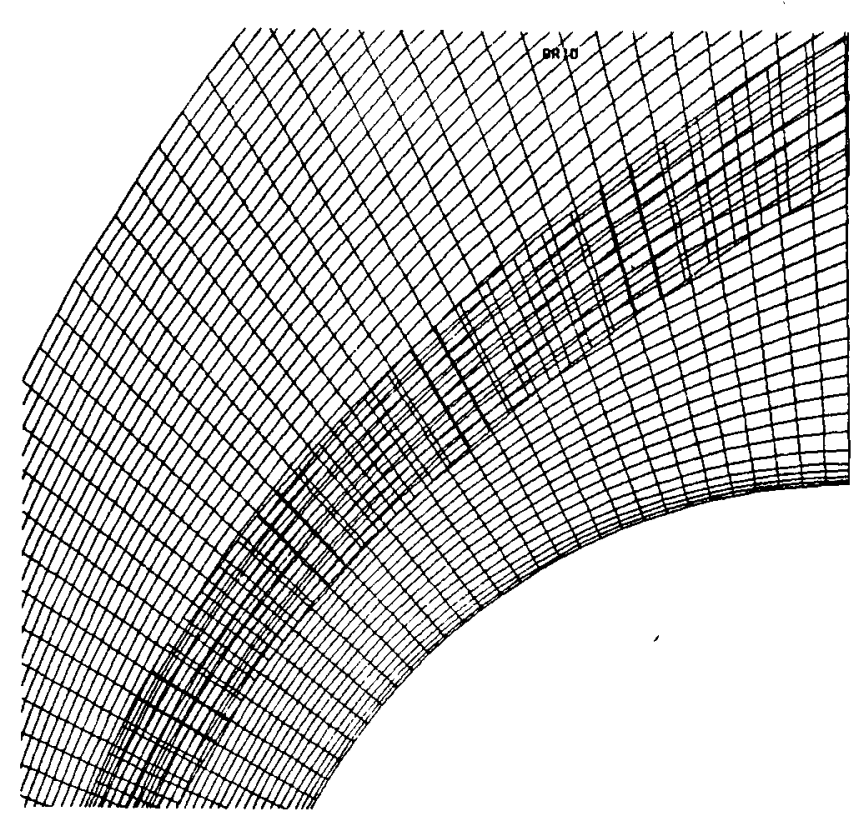

Fig. 11 (b) Close-up of region of overlap

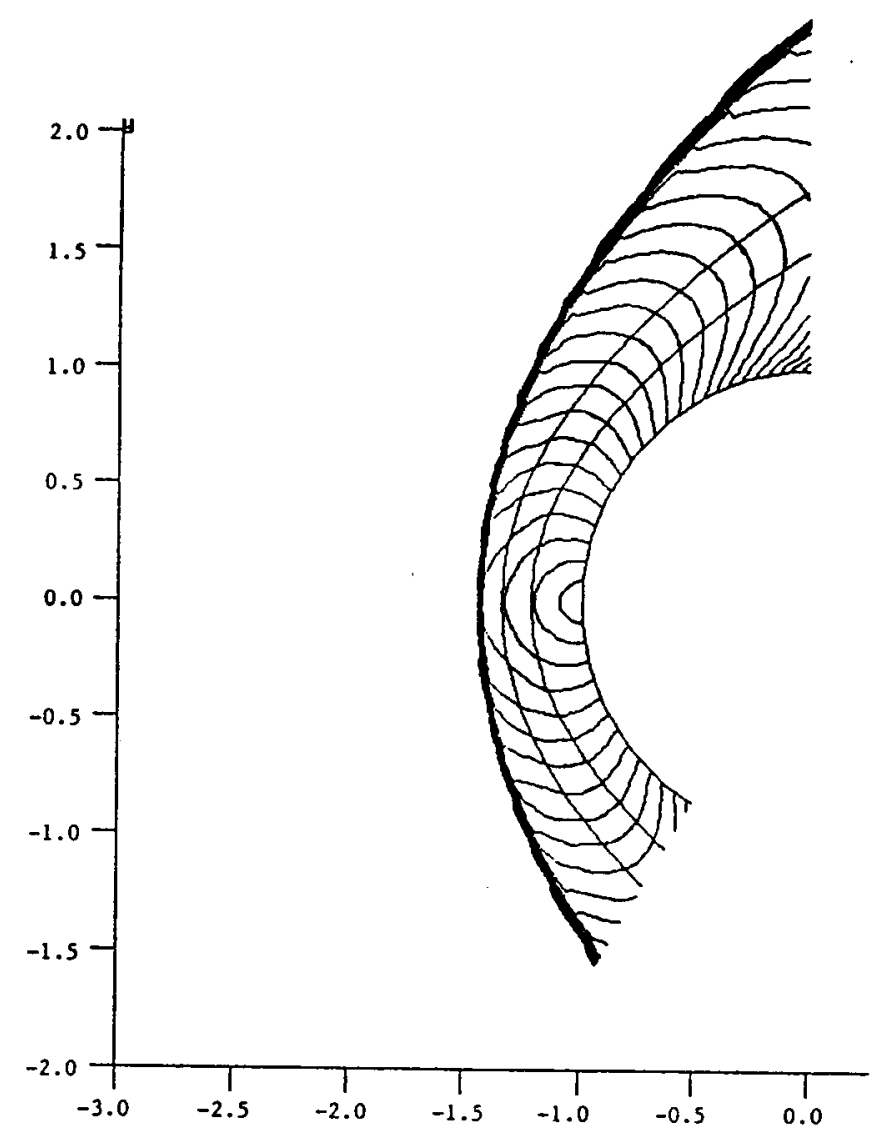

Fig. 11 (c) Mach number contours (zeroth order redistribution)

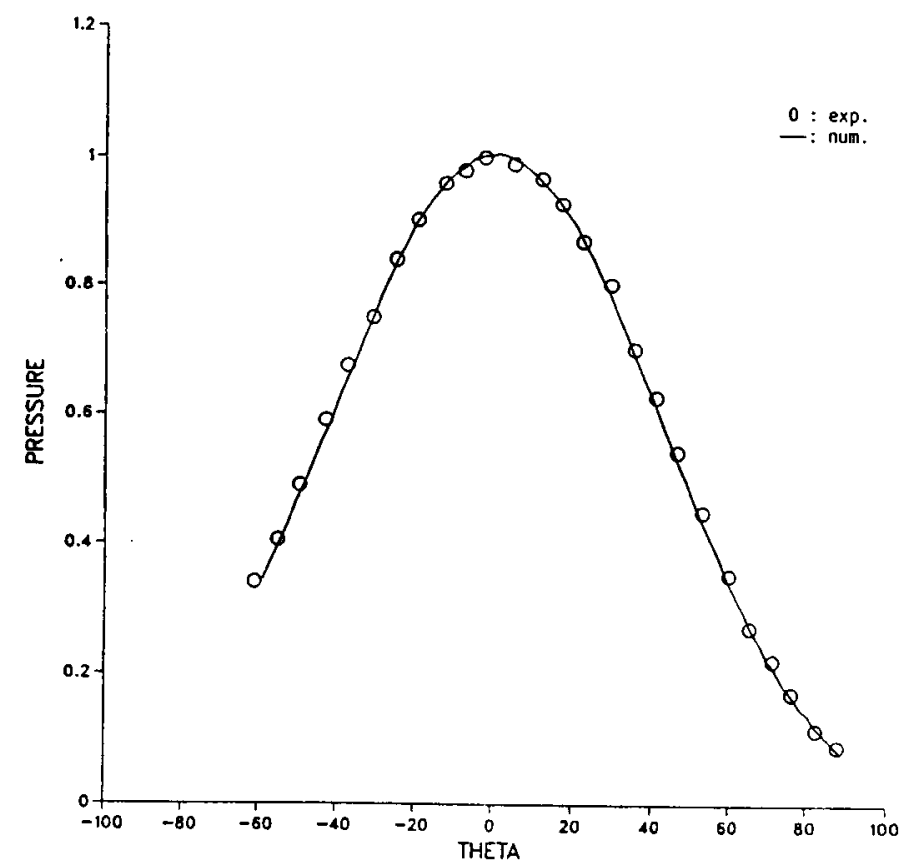

Fig. 11 (d) Pressure distribution along the cylinder (o:exp, 一:num) 


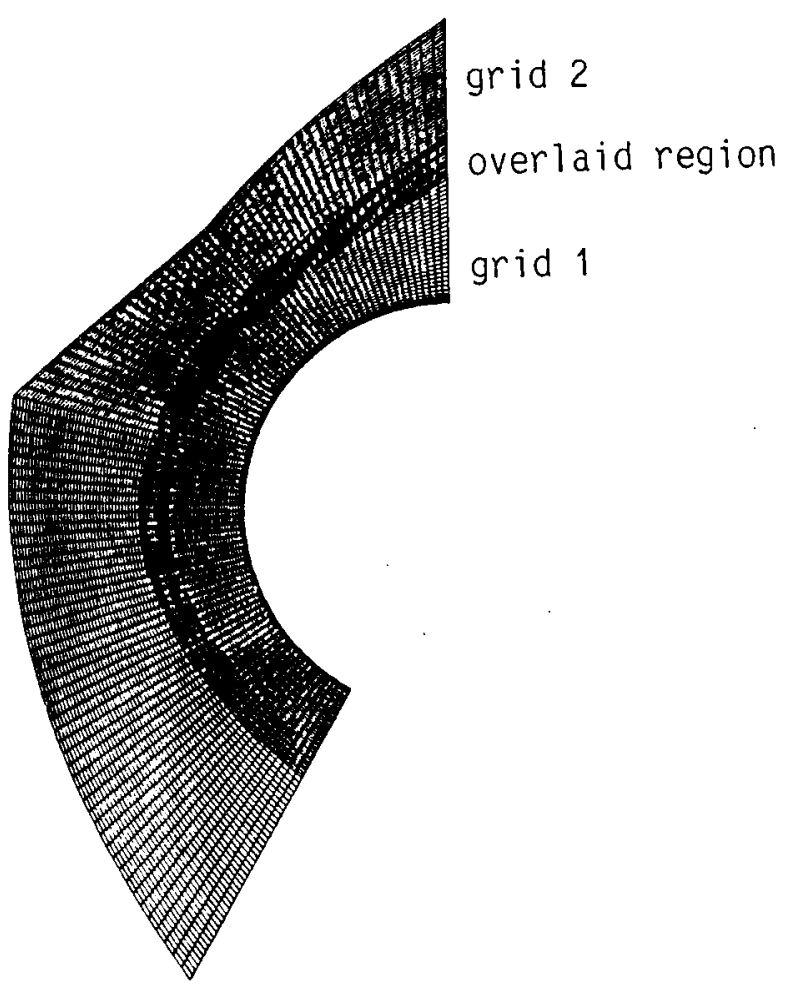

Fig. 12 (a) Shock-on-shock interaction: grid $1(89 \times 39)$, grid $2(99 \times 49)$

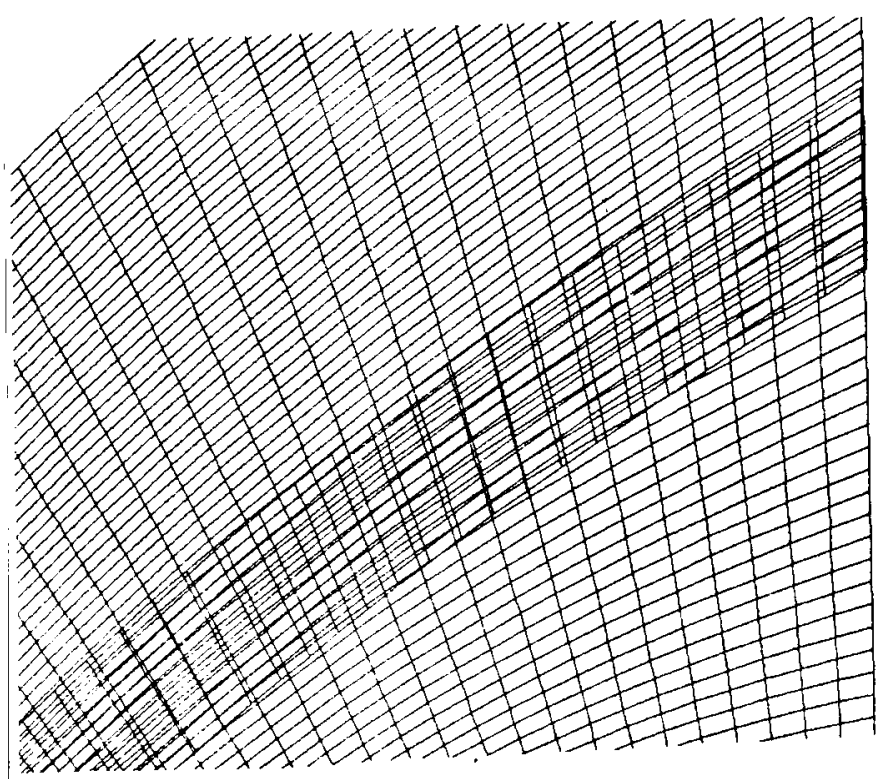

Fig. 12 (b) Close-up of region of overlap

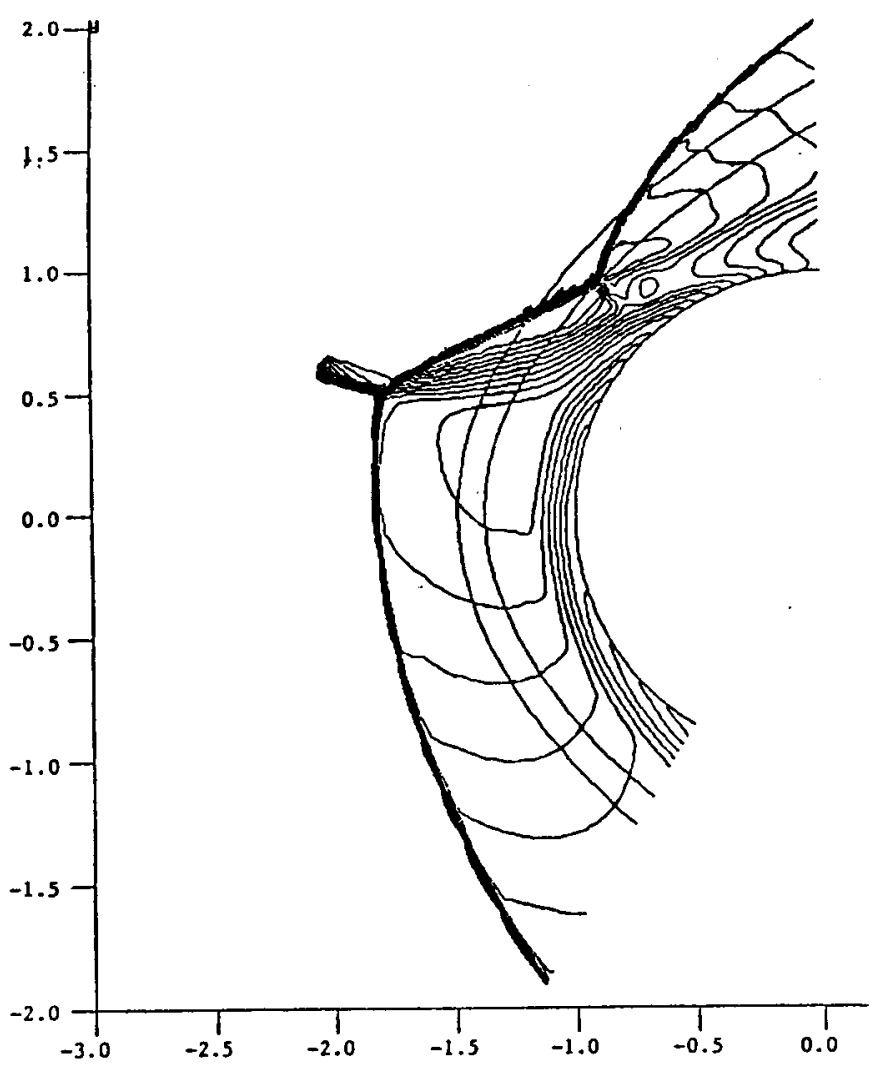

Fig. 12 (c) Mach number contours (zeroth order redistribution)

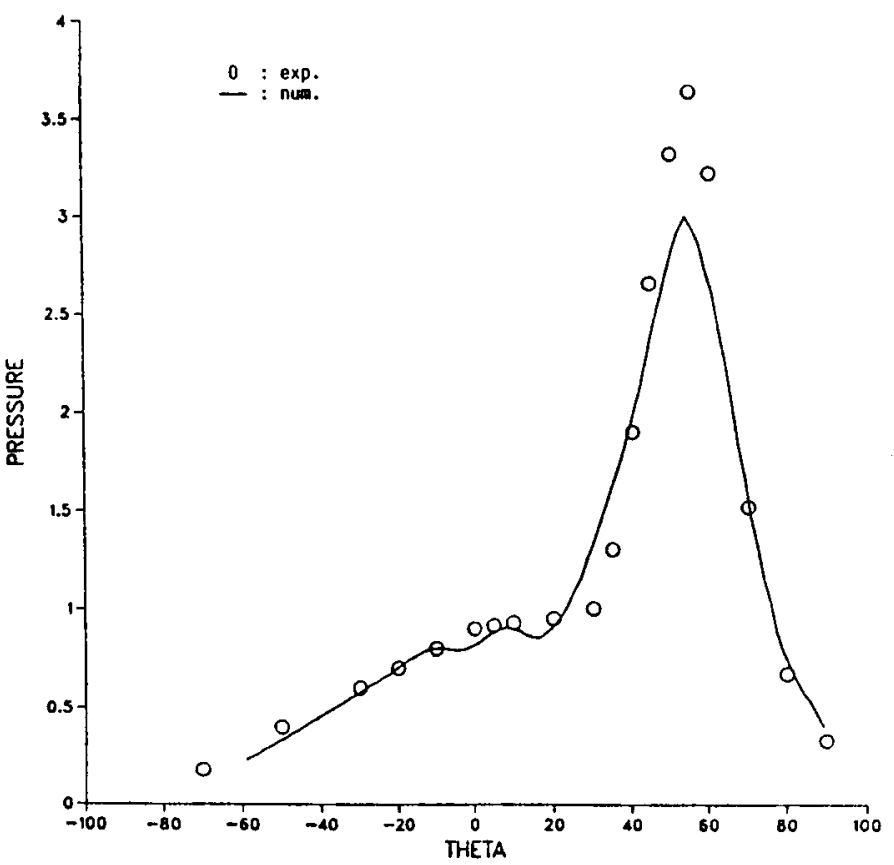

Fig. 12 (d) Pressure distribution along the cylinder (o:exp, 一:num) 


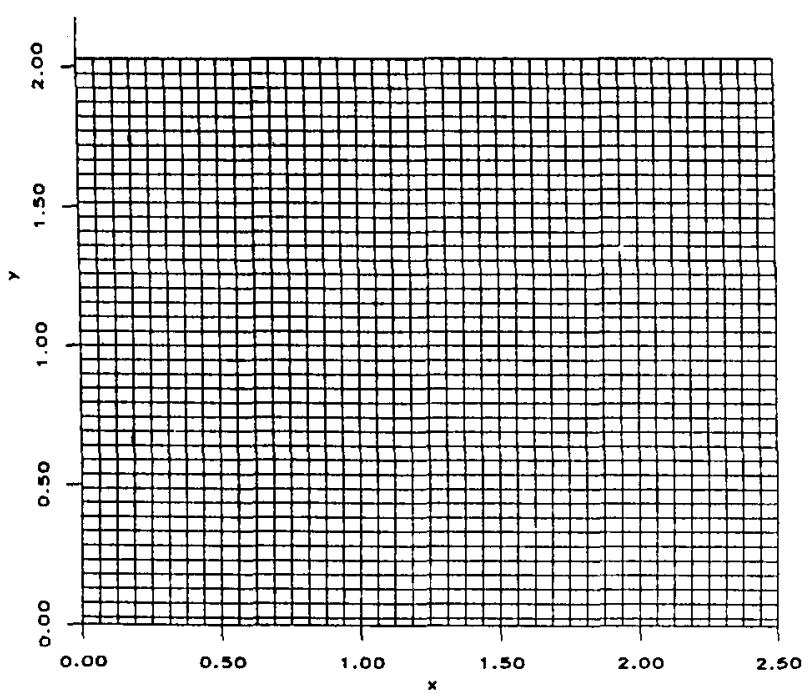

Fig. 13 (a) Multi-level grid adaptation: Base grid $(41 \times 41)$

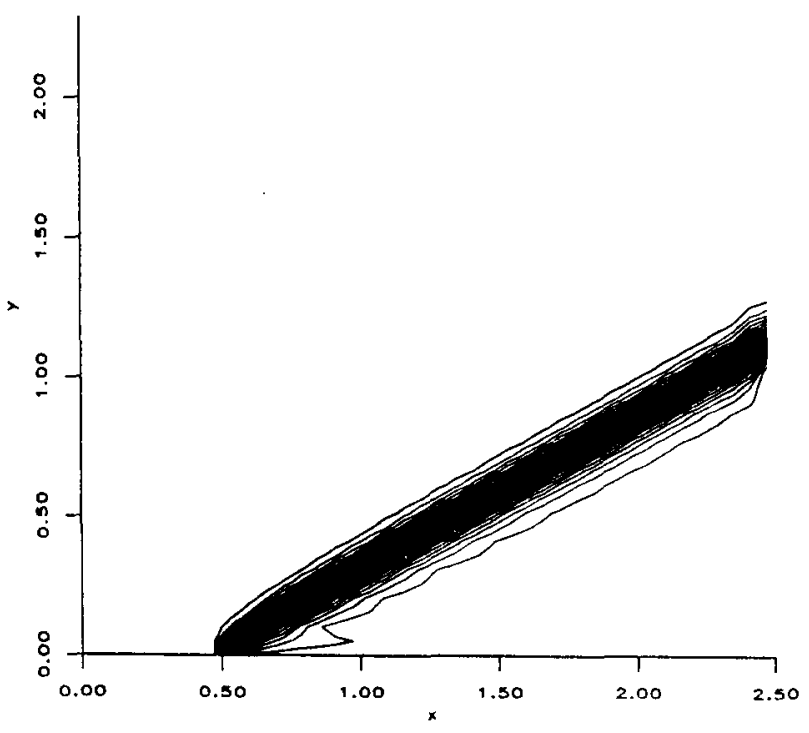

Fig. 13 (b) Pressure contours of an oblique shock

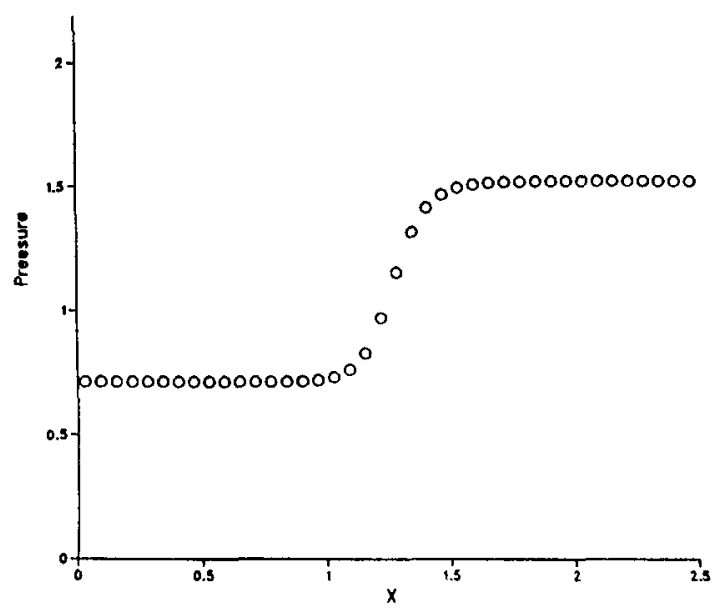

Fig. 13 (c) Pressure distribution at $y=0.5$

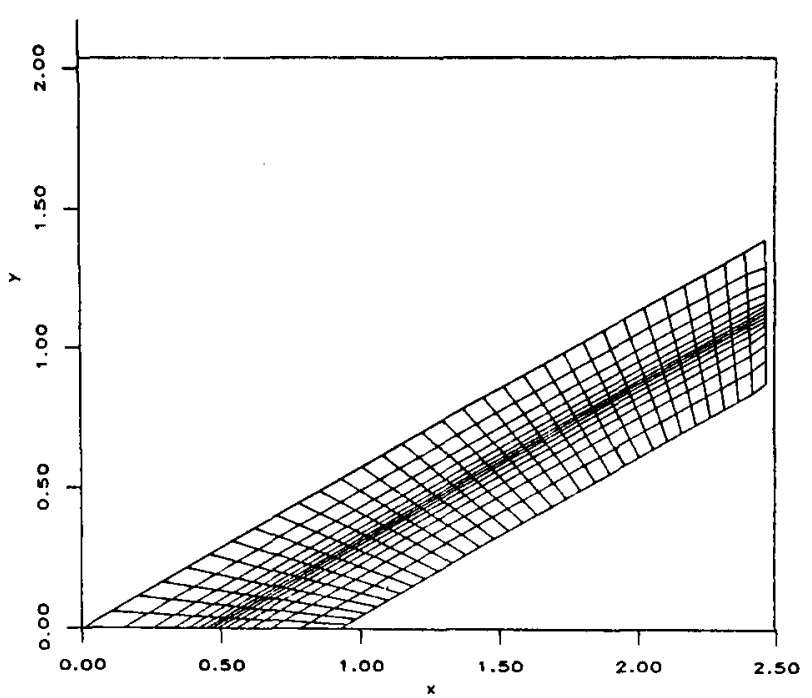

Fig. 14 (a) Next level adapted grid (33x15)

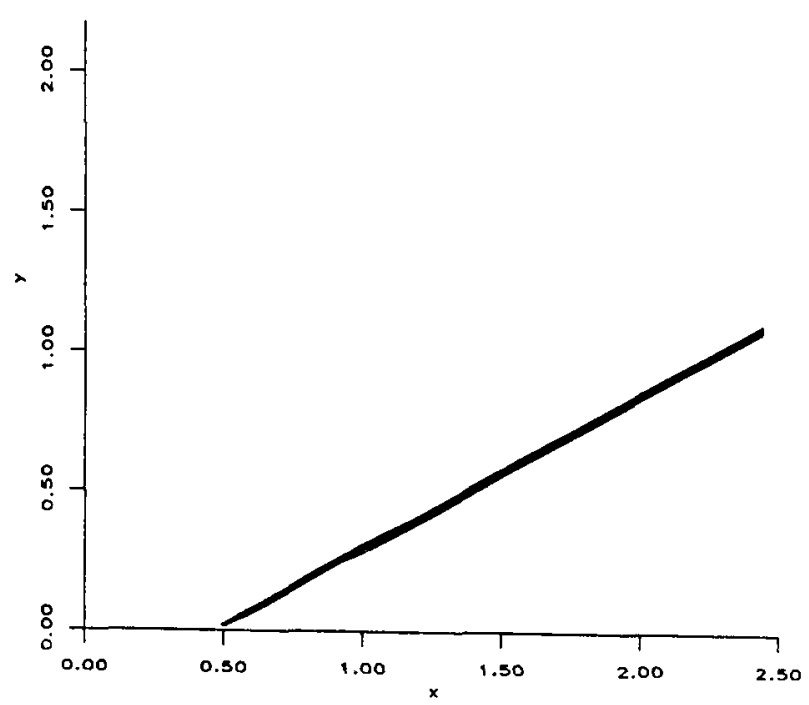

Fig. 14 (b) Pressure contours of an oblique shock on the adapted grid

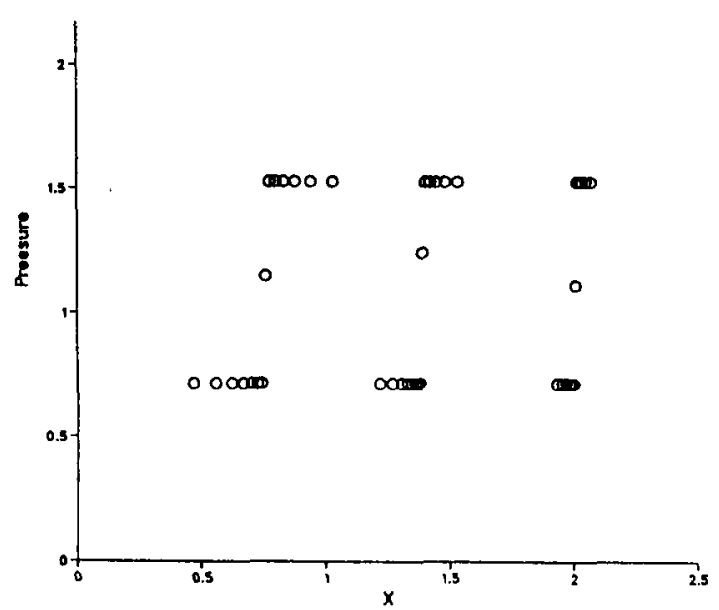

Fig. 14 (c) Pressure distributions at three different locations 


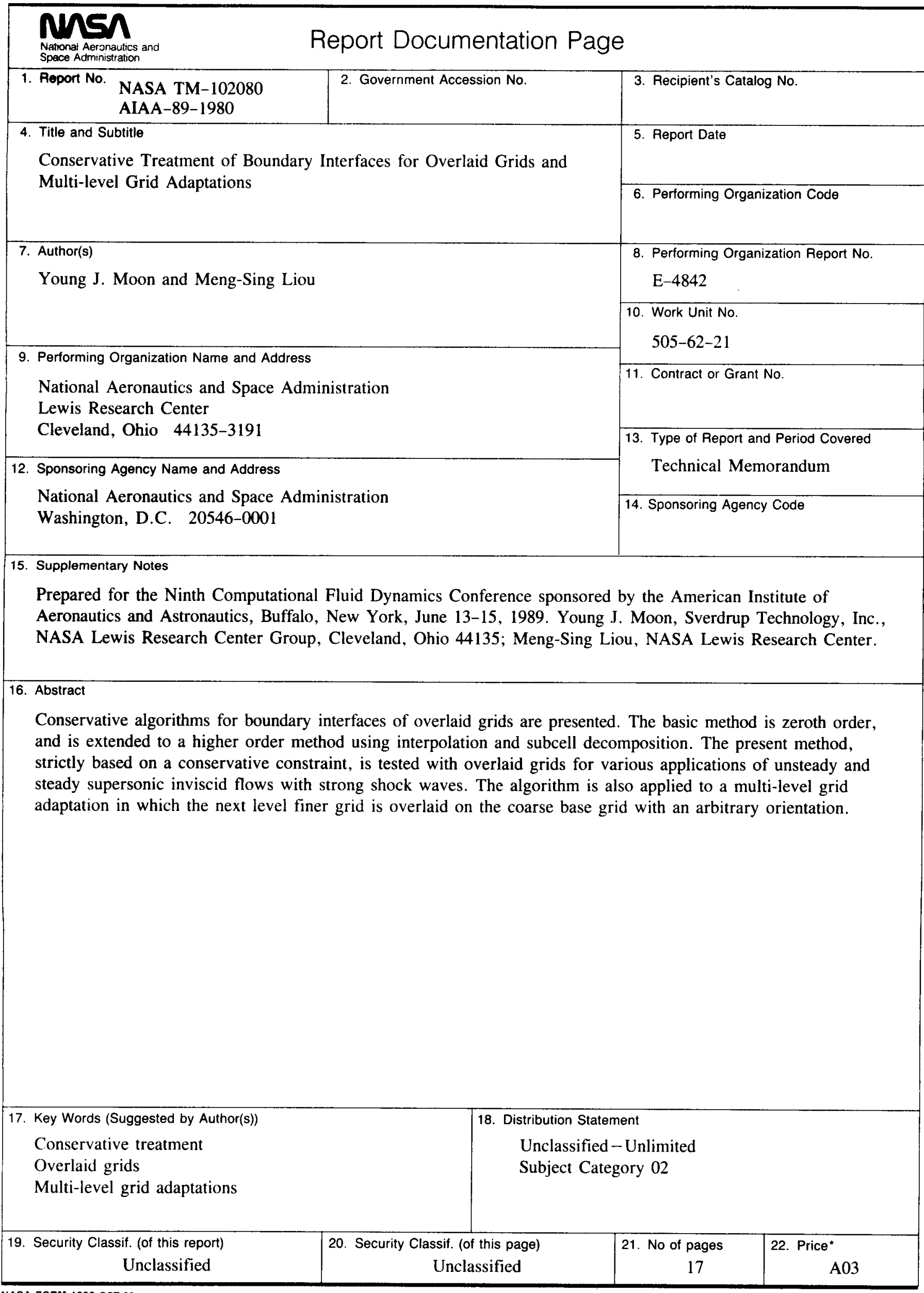

